\title{
Dense Periodic Packings of Tori
}

\author{
Ruggero Gabbrielli* \\ Interdisciplinary Laboratory for Computational Science, \\ Department of Physics, University of Trento, 38123 Trento, Italy \\ Yang Jiadt \\ Materials Science and Engineering Program, \\ School for Engineering of Matter, Transport and Energy, \\ Arizona State University, Tempe, Arizona 85281, USA \\ Salvatore Torquatd \\ Department of Chemistry, Department of Physics, \\ Program in Applied and Computational Mathematics, and \\ Princeton Institute for the Science and Technology of Materials, \\ Princeton University, Princeton, New Jersey 08544, USA
}

(Dated: February 28, 2014) 


\begin{abstract}
Dense packings of nonoverlapping bodies in three-dimensional Euclidean space $\mathbb{R}^{3}$ are useful models of the structure of a variety of many-particle systems that arise in the physical and biological sciences. Here we investigate the packing behavior of congruent ring tori in $\mathbb{R}^{3}$, which are multiply connected non-convex bodies of genus one, as well as horn and spindle tori. Specifically, we analytically construct a family of dense periodic packings of unlinked, congruent tori guided by the organizing principles originally devised for simply connected solid bodies [Torquato and Jiao, Phys. Rev. E 86, 011102 (2012)]. We find that the horn tori as well as certain spindle and ring tori can achieve a packing density not only higher than that of spheres (i.e., $\pi / \sqrt{18}=0.7404 \ldots$ ) but also higher than the densest known ellipsoid packings (i.e., $0.7707 \ldots$... In addition, we study dense packings of clusters of pair-linked ring tori (i.e., Hopf links), which can possess much higher densities than corresponding packings consisting of unlinked tori.
\end{abstract}

PACS numbers: $61.50 . \mathrm{Ah}, 05.20 . \mathrm{Jj}$

*Electronic address: ruggero.gabbrielli@unitn.it

$\dagger$ Electronic address: yang.jiao.2@asu.edu

${ }^{\ddagger}$ Electronic address: torquato@electron.princeton.edu 


\section{INTRODUCTION}

Dense packings of nonoverlapping bodies (i.e., hard particles) are useful models of a variety of low-temperature state of matter [1 6], granular media [4, 7], heterogeneous materials [4], and biological systems [8 10]. Probing the symmetries and other mathematical properties of the densest packings is a problem of interest in discrete geometry and number theory [11, 12]. In general, a packing is defined as a large collection of nonoverlapping solid objects in $d$-dimensional Euclidean space $\mathbb{R}^{d}$. Associated with a packing is the packing fraction (or density) $\phi$ defined as the fraction of space $\mathbb{R}^{d}$ covered by the particles.

The densest packings of nonoverlapping objects are of particular interest because of their relationship to ground states of matter and because such configurations determine the highdensity phases in the equilibrium phase diagram of hard-particle systems [13]. The determination of the densest packing arrangements of particles that do not fill all of space [14] is a notoriously challenging problem. For example, the rigorous proof of the conjecture for the densest arrangements of congruent (i.e., equal-sized) spheres put forth by Kepler [15], the most elementary non-space-filling shape, took almost four centuries. The conjecture, now a theorem, states that the packing fraction of spheres in three-dimensional Euclidean space can not exceed the value $\phi=\pi / \sqrt{18}=0.7404 \ldots$ [16]. There has been recent progress on the determination of the densest known packings of spheres of two different sizes [17], but there are no rigorous proofs that any of these packings are indeed the densest for a given size ratio and relative concentration.

The preponderance of studies of dense packings of nonspherical shapes in $\mathbb{R}^{3}$ have dealt with convex bodies, which are always simply connected and thus topologically equivalent to a sphere. Examples of such convex shapes, which are of genus zero, include ellipsoids [18], superballs [19, 20], cones [21], spherocylinders [22], lens-shaped particles [22], and various polyhedra [23 29]. More recently, researchers have found dense packings of nonconvex (or concave), simply connected bodies, such as non-convex superballs (including three-dimensional crosses) [19], dumbbell-shaped particles [30], certain non-convex polyhedra (including Szilassi polyhedra, tetrapods, octapods, and stellated dodecahedra) [29], and non-convex building blocks composed of clusters of nonspherical shapes [22]. Organizing principles and conjectures have been proposed that enable one to predict dense packing arrangements of simply connected bodies based on the characteristics of the particle shapes 
(e.g., symmetry, principal axes, and convexity) [22]. This includes a Kepler-like conjecture for the densest packings of the centrally-symmetric Platonic and Archimedean solids [25]. However, much less is known about dense packings of multiply connected solid bodies.

The focus of this paper is the determination of dense packings of congruent tori. A ring torus is an elementary multiply connected non-convex non-space-filling body of genus one, which constitutes an infinite variety of shapes [31]. The interest in such a family of shapes is not merely theoretical but has practical applications. One relevant example is in pharmaceutical technologies. Toroidal particles have been and are currently being investigated for use in drug delivery as they possess a higher surface area per unit volume than commonly used simply connected shapes such as short cylindrical-like pills [32]. Fabrication methods have proven successful either via colloidal crystallization [33] or microfluidics [34].

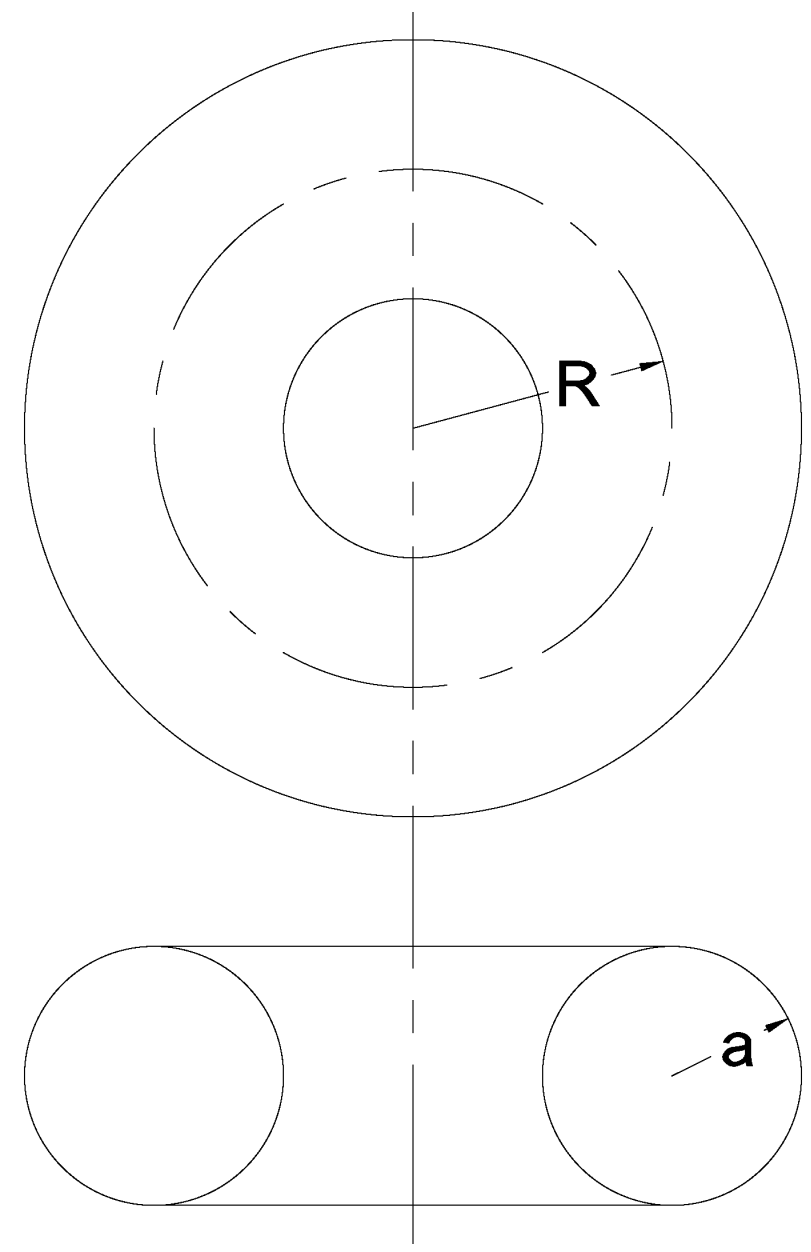

FIG. 1: The two radii defining a torus: major radius $R$ and minor radius $a$. We denote the major-to-minor radii ratio by $\gamma=R / a$. 
A torus in $\mathbb{R}^{3}$ is defined by the surface of revolution generated by revolving a circle in three dimensions about an axis that is coplanar with the circle (see Fig. 1). The surface of a torus centered at the origin can be written in parametric form as:

$$
\left\{\begin{array}{l}
x=(R+a \cos \phi) \cos \theta \\
y=(R+a \cos \phi) \sin \theta \\
z=a \sin \phi
\end{array}\right.
$$

where $0<\phi, \theta \leq 2 \pi$ and $R$ is the distance from the center of the generator circle to the axis of revolution or the major radius and $a$ is the radius of the circle itself or the minor radius. A torus can take a continuous range of shapes and is parametrized by the ratio

$$
\gamma=\frac{R}{a}
$$

where $R$ is the major radius and $a$ is the minor radius. In particular, tori are classified into three different types based on the value of their major-to-minor radii ratio (or radii ratio for short). For $\gamma<1$, the surface self-intersects at two points, thus generating a spindle torus. By construction, there is a region inside a spindle torus whose volume should be formally counted twice. This region has the shape of an American football and it is called a lemon [Fig. 2(f)]. In this work we only consider the external surface of the spindle torus, also known as the apple [Fig. 2(c)]. For $\gamma=1$ the torus surface is tangent to the torus axis. The surface normal is defined everywhere apart from a single point, the torus center. This is called a horn torus. For $\gamma>1$, the solid body is a ring torus. The surface normal is defined everywhere on the manifolds in such cases. Figure 2 shows these three different types of tori and their corresponding cut-aways that divide the tori in half.

If we substitute apples for spindle tori, the volume $v_{\mathrm{T}}$ and surface area $s_{\mathrm{T}}$ of such bodies are given by

$$
v_{\mathrm{T}}= \begin{cases}2 \pi\left[\frac{\left(2+\gamma^{2}\right)}{3} \sqrt{1-\gamma^{2}}+\gamma \cos ^{-1}(-\gamma)\right] \cdot a^{3}, & 0<\gamma<1, \\ 2 \pi^{2} \gamma \cdot a^{3}, & \gamma \geq 1,\end{cases}
$$

and

$$
s_{\mathrm{T}}= \begin{cases}4 \pi\left[\sqrt{1-\gamma^{2}}+\gamma \cos ^{-1}(-\gamma)\right] \cdot a^{2}, & 0<\gamma<1, \\ 4 \pi^{2} \gamma \cdot a^{2}, & \gamma \geq 1 .\end{cases}
$$




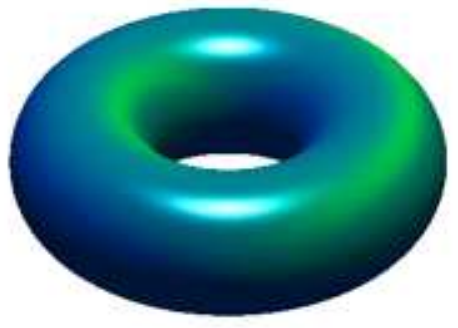

(a)

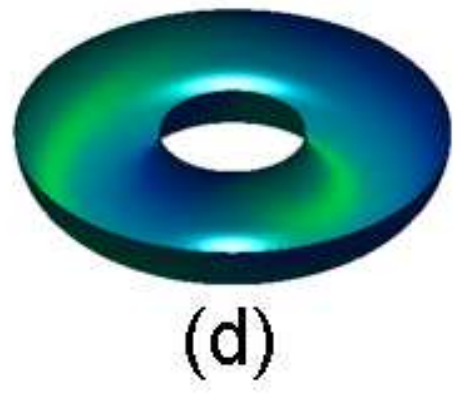

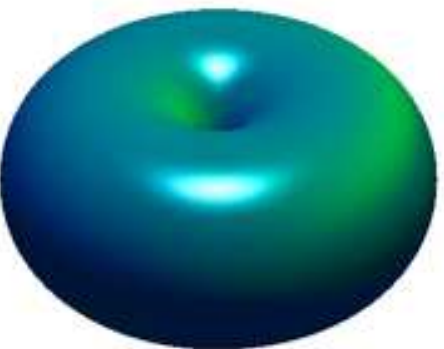

(b)

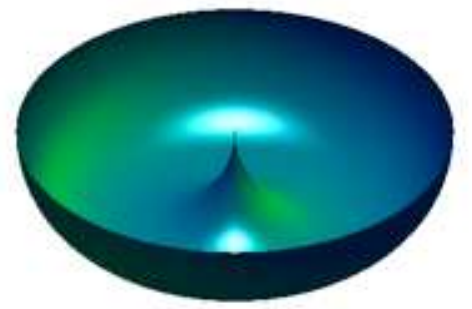

(e)
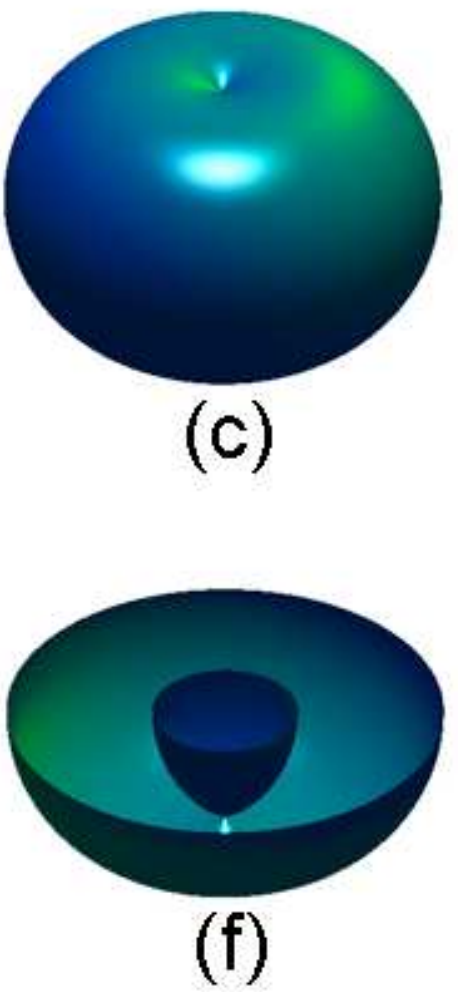

FIG. 2: Three different types of tori (upper panels) and the associated cut-aways that divide the tori in half (lower panels) as classified based on their radii ratio $\gamma$. (a) A ring torus with $\gamma>1$. (b) A horn torus with $\gamma=1$. (c) A spindle torus with $0<\gamma<1$. This is also the surface of an apple. (d) A cut-away of the ring torus shown in (a). (e) A cut-away of the horn torus shown in (b). (f) A cut-away of the spindle torus shown in (c). A half of a "lemon" is visible within.

The packing fraction $\phi$ of a configuration at number density $\rho$ (number of tori per unit volume) and lattice vectors $\mathbf{a}_{i}$ is simply given by

$$
\phi=\rho v_{\mathrm{T}} .
$$

In this paper, we analytically construct a family of dense periodic packings of unlinked ring tori characterized by their major-to-minor radii ratio $\gamma$. Such constructions are achieved by generalizing the organizing principles originally devised for simply connected nonspherical particles (both convex and non-convex) [22]. We find that for the horn tori $(\gamma=1)$, certain spindle $(\gamma<1)$ and ring tori $(\gamma>1)$, packings can be achieved with a density $\phi$ not only higher than that of spheres (i.e., $\pi / \sqrt{18}=0.7404 \ldots$ ) but also higher than the densest known ellipsoid packings (i.e., $0.7707 \ldots$... In addition, we study dense packings of clusters 
of certain linked tori, e.g., dimers composed of two tori with $\gamma=2$ linked in the simplest known form (i.e., a Hopf link).

The rest of the paper is organized as follows: In Sec. II, we present our analytical construction of a family of dense packings of tori with different radii ratios. In Sec. III, we construct dense packings of clusters of linked tori. In Sec. IV, we make concluding remarks.

\section{ANALYTICAL CONSTRUCTION OF A FAMILY OF DENSE PERIODIC PACKINGS OF TORI}

The general organizing principles proposed for simply connected solid objects suggest that when a centrally symmetric object is arranged on a Bravais lattice (one particle per fundamental cell), the number of contacts that the particle makes with its neighbors can be maximized, which leads to a dense packing [22]. If the object itself does not possess central symmetry, usually a centrally symmetric cluster composed of an even number of the individual objects can be constructed, whose Bravais-lattice packing provides a dense packing of the solid. However, the Bravais-lattice packing of the clusters are now a (nonBravais lattice) periodic packing of the original solid object, since there are multiple objects per fundamental cell. Since a torus is a centrally symmetric object, we first consider Bravais lattice packings of unlinked tori.

Vertically stacking tori exactly on top of each other to make infinitely large "cylindricallike" clusters, each of which are then placed on the sites of a triangular lattice, is an intuitively appealing configuration that one may wish to start with. In any cylindrical-like cluster, each torus contacts two neighbors, one above and the other below. Two tori contact one another through a common circle [see Fig. 3(a)]. When the cylindrical-like clusters are arranged on a triangular lattice, each torus in such a configuration is in contact with 8 neighbors, two of which share a circle and the remaining six are in contact with a single point lying on the equator [see Fig. 3(b)].

We note that a denser packing is obtained by slightly shifting the cylindrical-like clusters in the triangular-lattice packing along the axis of the "cylinders" so that each torus comes into contact with four more neighbors (relative to the simple triangular lattice configuration), thus reaching a "contact number" equal to 12 [see Fig. 3(c)]. (Note that two contacts are also circles in this case.) The lattice vectors associated with the constructed packing for 


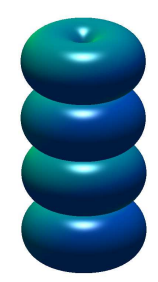

(a)

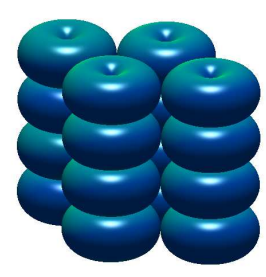

(b)

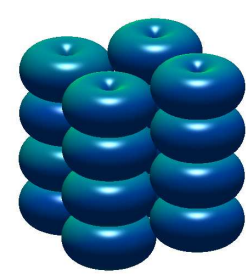

(c)

FIG. 3: Illustrations of dense periodic packings of tori. (a) A cylindrical-like cluster constructed by stacking tori exactly on top of one another. (b) A triangular-lattice packing of the cylindrical-like clusters of tori in which each torus has 8 contacting neighbors. (c) A dense periodic packing that improves upon the one shown in (b) with 12 contacting neighbors per torus.

congruent tori characterized by $R$ and $a$ are given by:

$$
\begin{gathered}
\mathbf{a}_{1}=[\gamma+1, \Gamma, 1] a, \\
\mathbf{a}_{2}=[-(\gamma+1), \Gamma, 1] a, \\
\mathbf{a}_{3}=\left[\begin{array}{ll}
0,0,2] a,
\end{array}\right.
\end{gathered}
$$

where:

$$
\Gamma=\sqrt{3 \gamma^{2}+2(2 \sqrt{3}-1) \gamma+2}
$$

The associated packing density is given by:

$$
\phi(\gamma)= \begin{cases}\pi \frac{\gamma \cos ^{-1}(-\gamma)+\frac{\left(2+\gamma^{2}\right) \sqrt{1-\gamma^{2}}}{3}}{2(1+\gamma) \Gamma} & 0<\gamma<1 \\ \frac{\pi^{2} \gamma}{2(1+\gamma) \Gamma} & \gamma \geq 1\end{cases}
$$

where $\gamma=R / a$ is the major-to-minor radii ratio of the tori. Fig. 4 shows the packing density as a function of radii ratio $\gamma$. In the case $\gamma=0$, the torus reduces to a sphere, which has the optimal packing density of $\pi / \sqrt{18}=0.7404 \ldots$ As $\gamma$ increases from 0 , an increase of packing density $\phi$ is immediately achieved. As the radii ratio increases, the packing density eventually begins to decrease due to the emergence of a large void region in the middle of the cylindrical-like clusters. Nonetheless, a wide spectrum of tori with radii ratio $\gamma \in(0,1.47074 \ldots)$ can pack more densely than spheres in this construction.

Interestingly, a maximum packing density $\phi_{\max }=0.786322 \ldots$ is achieved by spindle tori of radii ratio $\gamma^{*}=0.820265 \ldots$. Note that a spindle torus has no holes and thus is a better packer than the ring tori. The density associated with the horn tori (with $\gamma=1$ ) is given 


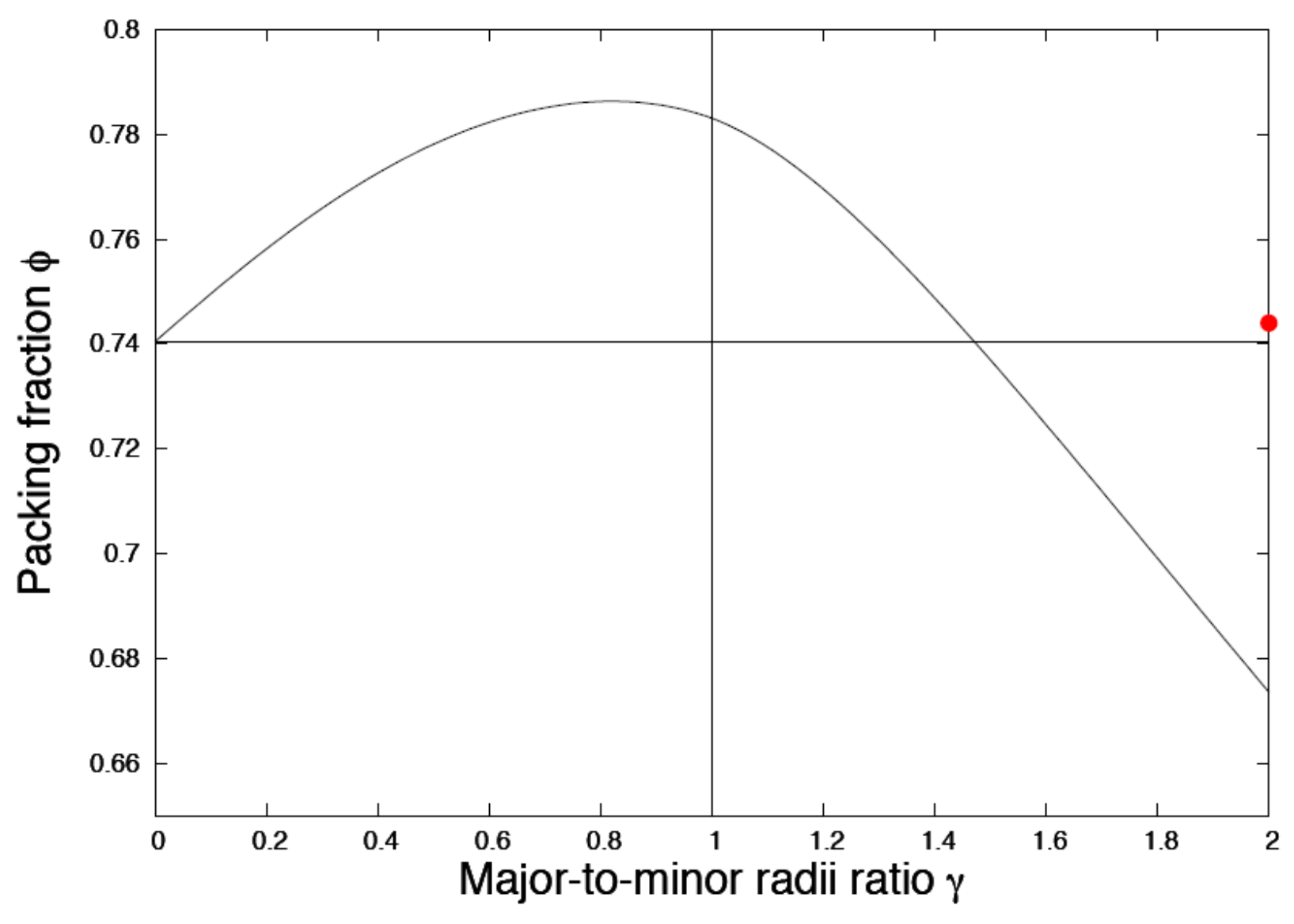

FIG. 4: Packing fraction $\phi$ of the periodic packing of tori shown in Fig. 2 as a function of the major-to-minor radii ratio $\gamma$. The packing fraction of the arrangement in Fig. [6 is also reported at the extreme value of $\gamma=2$ indicated in the graph as a filled-in circle.

by

$$
\phi(\gamma)=\phi(1)=\frac{\pi^{2}}{4 \sqrt{3+4 \sqrt{3}}}=0.783076 \ldots
$$

For ring tori, the maximum density becomes arbitrarily close to that of horn tori as the radii ratio approaches unity, i.e., $\gamma \rightarrow 1$.

\section{DENSE PACKINGS OF PAIR-LINKED TORI}

As the major-to-minor radii ratio $\gamma$ becomes large, the hole in a torus grows in size and makes the cylindrical-like arrangements inefficient packings. If we allow the tori to be linked (knotted) with one another, the void space in the torus hole can be used to improve the packing density. Mathematically, allowing the tori to link to determine the densest packings 
of tori among all possible configurations is perfectly valid given the fact that a torus has genus one.

First, we note that ring tori with $\gamma \geq 2$ can be assembled into Hopf links. A Hopf link here refers to pair-linked tori that are arranged to form a periodic. Then such a "dimer" can pair with an identical object to form a unit made of 4 tori; see Fig. 5. Based on our general organizing principles, a dense of packing of such a centrally symmetric 4-tori cluster can be achieved by a Bravais-lattice packing of the clusters, which we describe below.

Different views of this packing arrangement are shown in Fig. 6, Lattice vectors and packing fraction can be computed by solving the following system of transcendental equations:

$$
\left\{\begin{array}{l}
\sin \beta=\frac{2-\sin \delta^{\prime}}{2+\cos \beta^{\prime}} \\
\sin \delta=\frac{1-\sin \beta^{\prime}}{2+\cos \delta^{\prime}} \\
\cos ^{2} \beta=\frac{\beta^{\prime}-1}{\delta-1} \\
\cos ^{2} \delta=\frac{\delta^{\prime}-1}{\beta-1} \\
x=\cos \beta\left(2+\cos \beta^{\prime}\right)+\cos \delta\left(2+\cos \delta^{\prime}\right)
\end{array}\right.
$$

which yields $\beta=0.5308 \ldots, \beta^{\prime}=0.4134 \ldots, \delta=0.2113 \ldots, \delta^{\prime}=0.5514 \ldots$ and $x=$ $5.3028 \ldots$... The corresponding lattice vectors are given by

$$
\begin{aligned}
\mathbf{a}_{1} & =[4,2,0] a, \\
\mathbf{a}_{2} & =[-2,4,0] a, \\
\mathbf{a}_{3} & =[1,3,2 x] a,
\end{aligned}
$$

and packing fraction is $\phi=0.7445 \ldots$. This significantly improves up on the density associated with the periodic packing of unlinked tori based on cylindrical-like clusters and is even higher than the optimal sphere packing fraction.

Tori of radii ratio larger than 2 (i.e., $\gamma>2$ ) can be locally packed into a dense interlocked cluster. For example, Fig. 7(a) shows a cluster of 5 interlocked tori with 4-fold symmetry. Fig. [(b) shows a cluster of 6 interlocked tori, which resemble a single torus. Such an arrangement represents the most compact way to pack them into a larger torus. Its crosssection includes one circle surrounded by five toric sections. This leads to the possibility of constructing dense packings of ring tori with large radii ratios possessing self-similar properties. However, due to the complexity of the local packing arrangements, such constructions 


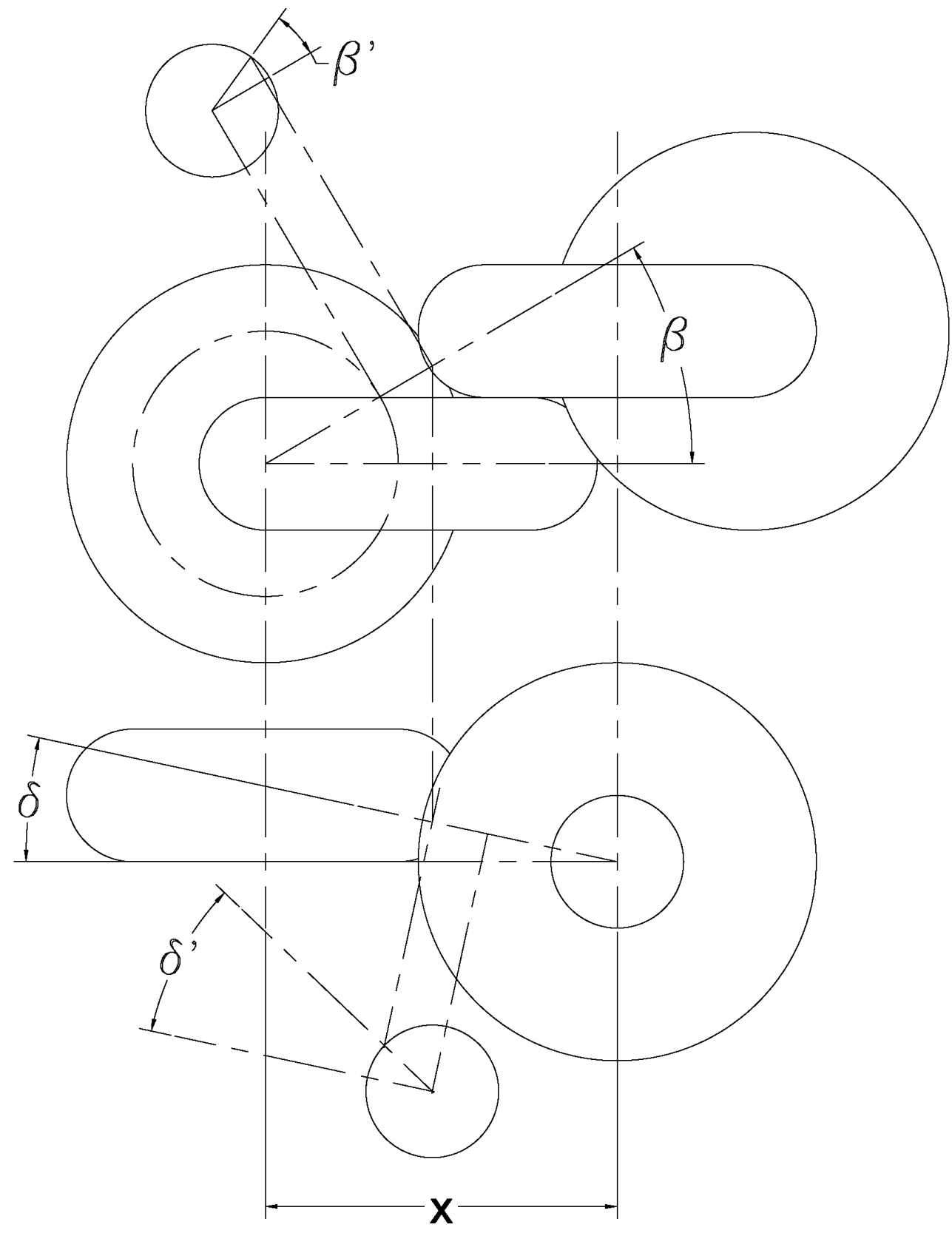

FIG. 5: Contact point between dimers in the densest known packing of tori of radii ratio 2. This sketch has been used for the determination of the only non-trivial lattice vector.

would involve nontrivial analytical treatments. 

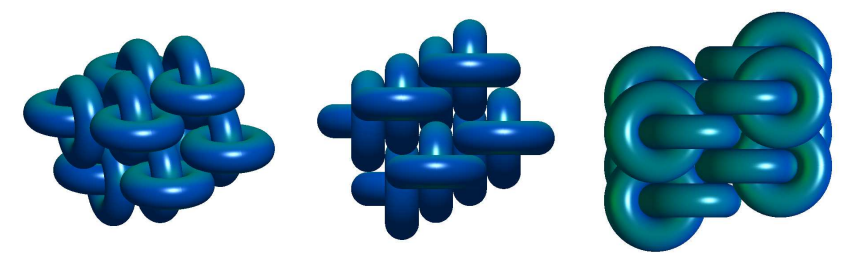

FIG. 6: The densest known packing of tori of radii ratio 2. Packing fraction is $\phi=0.7445 \ldots$ The images show four periodic units, each containing four tori.

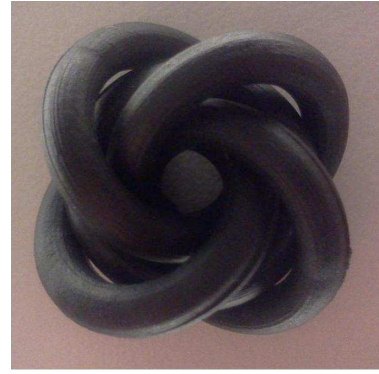

(a)

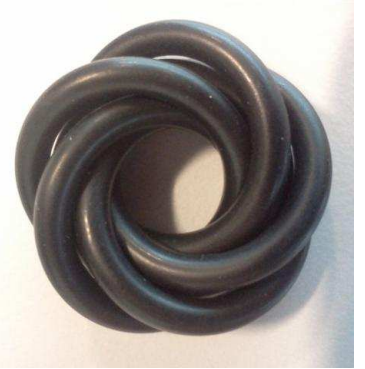

(b)

FIG. 7: (a) Image of a cluster of 5 interlocked elastomeric O-rings with 4-fold symmetry. (b) Image of a bundle of 6 interlocked O-rings. Such an arrangement represents the most compact way to pack them into a larger torus. Its cross-section includes one circle surrounded by five toric sections.

\section{CONCLUSIONS}

In this paper, we have reported analytical constructions of dense packings of tori in $\mathbb{R}^{3}$, which are elementary examples of multiply connected solids of genus one. Specifically, we have obtained a family of periodic packings of unlinked, congruent tori with a wide range of radii ratios. In this family, the packings of tori with radii ratio $\gamma \in(0,1.7408 \ldots)$ possess a higher density $\phi$ than the optimal sphere packing density $\pi / \sqrt{18}=0.7404 \ldots$ In other words, all spindle tori and the horn tori pack more densely than spheres; and even certain ring tori can pack more densely than spheres. A maximum packing density $\phi_{\max }=0.7863 \ldots$ is achieved by spindle tori of radii ratio $\gamma^{*}=0.8203 \ldots$

For ring tori with large radii ratios, the large void space in the holes of the tori eventually makes them bad packers. However, one can take advantage of the holes by considering linked or interlocked clusters of tori to achieve higher packing densities. For example, when two ring tori with $\gamma=2$ are arranged in a Hopf link dimer with central symmetry, a very dense lattice packing can be obtained. This indicates that the organizing principles originally 
formulated for simply connected convex and non-convex solid bodies [22] also apply to these more complex shapes.

Finally, we would like to note that for ring tori with very large radii ratios, more sophisticated packing arrangements could be identified that possess much higher packing densities than the simple periodic constructions based on the cylindrical-like stackings of the rings. However, such complex arrangements are difficult to find analytically. A recently developed

adaptive-shrinking-cell optimization procedure [35] could be used to search numerically for such complex packings of tori.

\section{Acknowledgments}

R.G. was supported by the Autonomous Province of Trento and the European Union's Seventh Framework Programme for research (COFUND - Call 1 - Postdoc 2010). Y. J. was supported by the start-up funds provided by Arizona State University. S.T. was supported in part by the Division of Mathematical Sciences at the National Science Foundation under Award No. DMS-1211087. This work was partially supported by a grant from the Simons Foundation (Grant No. 231015 to Salvatore Torquato).

[1] J. D. Bernal in Liquids: Structure, Properties, Solid Interactions (eds Hughel, T. J.) (Elsevier, New York, 1965).

[2] R. Zallen, The Physics of Amorphous Solids (Wiley, New York, 1983).

[3] P. M. Chaikin and T. C. Lubensky, Principles of Condensed Matter Physics (Cambridge University Press, Cambridge, 2000).

[4] S. Torquato, Random Heterogeneous Materials: Microstructure and Macroscopic Properties (Springer-Verlag, New York, 2002).

[5] M. J. Pond, J. R. Errington, T. M. Truskett, J. Chem. Phys. 135, 124513 (2011).

[6] M. Marechal, M. Hermes, and M. Dijkstra, J. Chem. Phys. 135, 034510 (2011).

[7] S. F. Edwards, in Granular Matter (eds Mehta, A.) p121 (Springer-Verlag, New York, 1994).

[8] Liang, J. and Dill, K. A., Biophys J. 81, 751 (2001).

[9] P. K. Purohit, J. Kondev, and R. Phillips, Proc. Natl. Acad. Sci. 100, 3173 (2003). 
[10] Y. Jiao and S. Torquato, Phys. Biol. 9, 036009 (2012).

[11] J. H. Conway and N. J. A. Sloane, Sphere Packings, Lattices and Groups (Springer, New York, 1998).

[12] H. Cohn and N. Elkies, Ann. Math. 157, 689 (2003).

[13] S. Torquato and F. H. Stillinger, Rev. Mod. Phys. 82, 2633 (2010).

[14] R. Gabbrielli, Y. Jiao, and S. Torquato, Phys. Rev. E 86, 041141 (2012).

[15] J. Kepler, Strena seu de nive sexangula (1611).

[16] T. C. Hales, Ann. Math. 2, 1065 (2005).

[17] A. B. Hopkins, Y. Jiao, F. H. Stillinger, and S. Torquato, Phys. Rev. Lett. 107, 125501 (2011);

A. B. Hopkins, F. H. Stillinger, and S. Torquato, Phys. Rev. E 85, 021130 (2012).

[18] A. Donev, F. H. Stillinger, P. M. Chaikin, and S. Torquato, Phys. Rev. Lett. 92, 255506 (2004).

[19] Y. Jiao, F. H. Stillinger, and S. Torquato, Phys. Rev. E 79, 041309 (2009).

[20] R. Ni, A. P. Gantapara, J. de Graaf, R. van Roij, and M. Dijkstra, Soft Matter 8, 8826 (2012).

[21] J. V. Whitworth, Proc. London Math. Soc. 53, 422 (1951).

[22] S. Torquato and Y. Jiao, Phys. Rev. E 86, 011102 (2012).

[23] E. R. Chen, M. Engel, and S. C. Glotzer, Discr. \& Comput. Geom. 44, 253 (2010).

[24] U. Betke and M. Henk, Comput. Geom. 16, 157 (2000).

[25] S. Torquato and Y. Jiao, Nature 460, 876 (2009).

[26] Y. Kallus, V. Elser, S. Gravel, Discrete Comput. Geo. 44, 245 (2010).

[27] Y. Jiao and S. Torquato, J. Chem. Phys. 135, 151101 (2011).

[28] P. F. Damasceno, M. Engel, and S. C. Glotzer, Science 337, 453 (2012).

[29] J. de Graaf, R. van Roij, and M. Dijkstra, Phys. Rev. Lett. 107, 155501 (2011).

[30] J. D. Forster, J.-Gyu Park, M. Mittal, H. Noh, C. F. Schreck, C. S. O’Hern, H. Cao, E. M. Furst, and E. R. Dufresne, ACS Nano 5, 6695 (2011).

[31] We note that another elementary example of a multiply connected non-convex body is a sphere in which a very thin cylinder passing through the sphere center is removed. Such a modified sphere is topologically equivalent to a torus, but the packing fraction of configurations of such genus-one objects can never exceed that of the solid sphere, unlike packings of tori. This clearly illustrates the importance of the geometry of the particle, not just the topology, in determining its packing behavior. 
[32] S. Ungphaiboon, D. Attia, G. Gomez d'Ayala, P. San- songsak, F. Cellesi, and N. Tirelli, Soft Matter 6, 4070 (2010).

[33] O. D. Velev, A. M. Lenhoff, and E. W. Kaler, Science 287, 2240 (2000).

[34] Y. Wang, J. Mater. Chem. 39, 179 (2007).

[35] S. Torquato and Y. Jiao, Phys. Rev. E 80, 041104 (2009). 